\title{
Rate of Satisfaction of Veterans with Supplementary Insurance and Their Families from Inpatient Services
}

\section{ART ICLE INF O}

\section{Article Type}

Descriptive Study

\section{Authors}

Maftoon F. ${ }^{1} P h D$

Mousavi B.* $M D, M P H$,

Soroush M.R. ${ }^{2} M D, M P H$

Mohammad K. ${ }^{3} P h D$

Rahimpoor D. ${ }^{2} M D$,

Khobyaryan A. ${ }^{2} M D$

How to cite this article
Maftoon F, Mousavi B, Soroush M
R, Mohammad K, Rahimpoor D,
Khobyaryan A. Rate of Satisfaction
of Veterans with Supplementary
Insurance and Their Families fr-
om Inpatient Services. Iranian Jo-
urnal of War \& Public Health.
2017;9(4):211-216.

*Janbazan Medical and Engineering Research Center (JMERC), Tehran, Iran

${ }^{1}$ Health Metrics Research Center, Institute for Health Sciences Research, Tehran, Iran

${ }^{2}$ Janbazan Medical and Engineering Research Center (JMERC), Tehran, Iran

${ }^{3}$ Epidemiology \& Biostatistics Department, Health Faculty, Tehran University of Medical Sciences, Tehran, Iran

\section{Correspondence}

Address: No 17, Janbazan Medical and Engineering Research Center (JMERC), Farokh Street, Mogadase Ardabili Street, Tehran, Iran Phone: +98 (21) 22416938 -

Fax: +98 (21) 22412550 mousavi.b@gmail.com

\section{Article History}

Received: February 6, 2017

Accepted: May 7, 2017

ePublished: November 6, 2017

\section{A B S T R A C T}

Aims The quality of healthcare services and the level of satisfaction with health services among veterans and their families are of particular importance in public health systems. The purpose of this study was to assess the rate of satisfaction of veterans with supplementary insurance and their families from hospital services.

Instruments \& Methods In this descriptive cross-sectional study, in 2015, the level of satisfaction of 210 veterans with supplementary insurance and their families in Martyrs and Sacrificers Foundation was evaluated by a researcher-made questionnaire. The participants were randomly selected. Data were analyzed by logistic regression test.

Findings The level of satisfaction with the process of inpatient service was $91.4 \%$, the satisfaction with the distance from hospital location was $79.5 \%$ and the satisfaction with the process of refunding the costs was 38\%. There was no significant relationship between age, gender, level of education, occupation, residency and status of sacrifice with distance from hospital location ( $\mathrm{p}>0.05)$.

Conclusion The satisfaction of veterans with supplementary insurance and their families, with hospital services is desirable. The rate of satisfaction with distance from hospital location is acceptable, but the satisfaction with the process of refunding the costs is low.

\section{Keywords Patient Satisfaction; Inpatients; Veterans; Hospital; Health Care Costs}

\section{CIT A T I O N L IN K S}

[1] Essentials of healthcare organization and ... [2] Assessment of Patients Satisfaction with Hospital Services in Razi ... [3] Satisfaction of child victims of landmines with services ... [4] Utilization Model of Health Services in Retirees ... [5] The role of participative management (suggestion system) in ... [6] Assessing satisfaction in health and long term care: Practical ... [7] Satisfying solutions? a review of some unresolved ... [8] Modeling patient satisfaction and service ... [9] Patient satisfaction with nursing care in the context ... [10] Patient satisfaction with health care: Critical outcome ... [11] Medical equipment and quality of life in ... [12] Satisfaction with outpatient visits in veterans covered by supplementary health ... [13] Doctor-patients communication and patient satisfaction ... [14] Process and outcomes in general practice consultations: Problems in defining high quality ... [15] Development and testing of a visit-specific patient satisfaction questionnaire: The Princess Margaret hospital satisfaction ... [16] The relationship of patient satisfaction with care and clinical ... [17] Care receivers satisfaction in family physician and referral system: a national ... [18] Survey of satisfaction of rural insured covered by the health insurance organization and providers in the referral implication ... [19] Satisfaction rate of hospitalized patients from the provision of health services in Shahid Beheshti and Yahya Nejad ... [20] Inpatient satisfaction: Medical services delivery in social security hospital ... [21] Satisfaction of janbazan and azadegan referring health center of janbazan ... [22] Health services satisfaction among chemical warfare survivors ... [23] Health care service satisfaction among chemical warfare survivors with severe ophthalmogic ... [24] Satisfaction with outpatient visits in veteranscovered ... [25] Satisfaction with supplemental health insurance and pharmaceutical care among veterans ... [26] Satisfaction of veterans from health services and their impact on mental ... [27] Satisfaction of martyrs' families of supplemental insurance in receiving ... [28] Satisfaction with outpatient health care services in ... [29] Patient Satisfaction of Female and Male Users of Veterans Health Administration ... [30] Satisfaction of supplementary insurance and medical ... 
بهداشت عمومى از بودجه دولت و ارايه آن از طريق سيستم

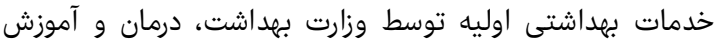

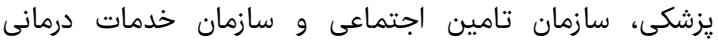

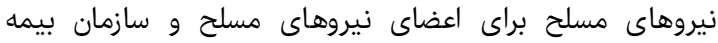

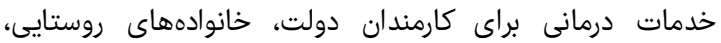

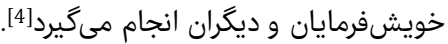

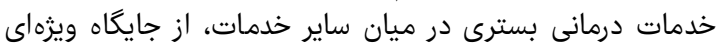

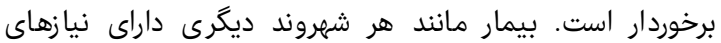

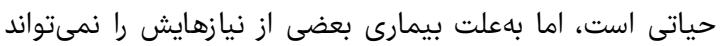

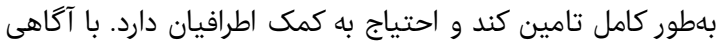

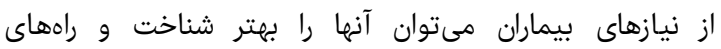

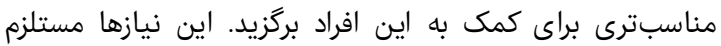

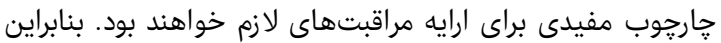

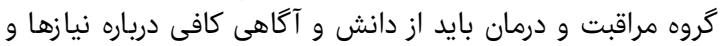

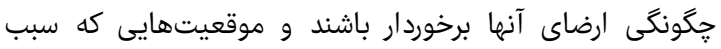

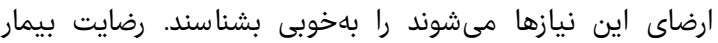

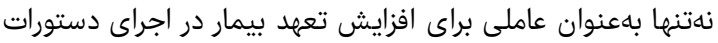

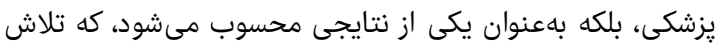

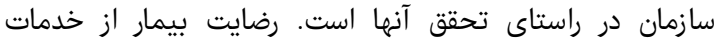

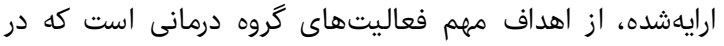

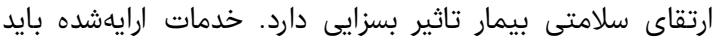

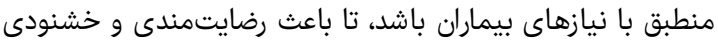

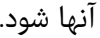

رضايت مشترى، ابزارى مهم در اندازمكيرى كيفيت خدمات درد

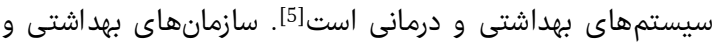

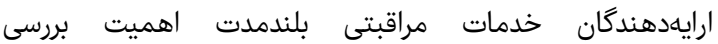

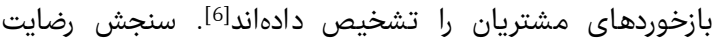

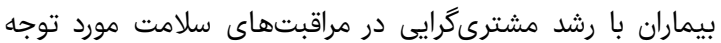

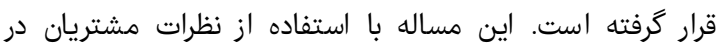

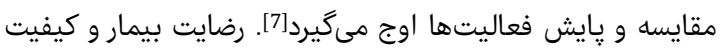

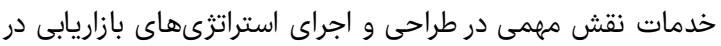

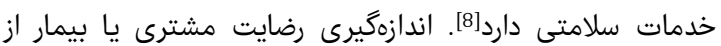

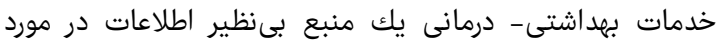

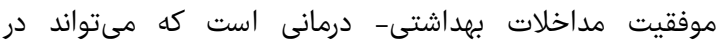
بيمارستان، مطب يا جامعه انجام شود. توجه به به مشترى در در حوزه

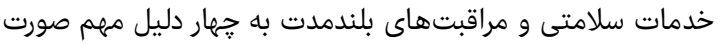

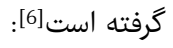

1- افزايش رقابت در ارايه خدمات سلامتى در نتيجه اجراى مراي

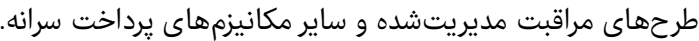

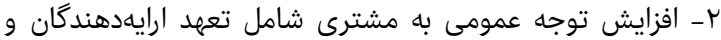

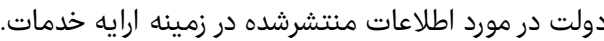

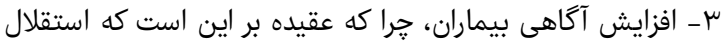

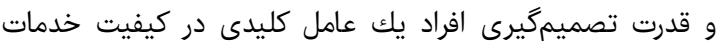

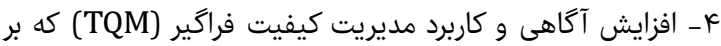

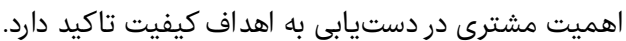

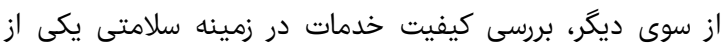

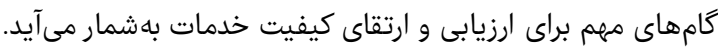

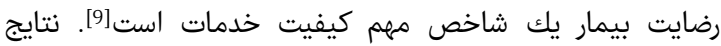

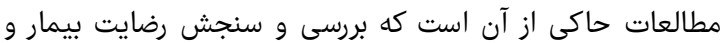

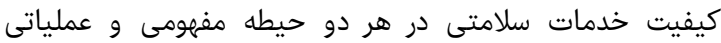

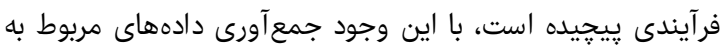

\section{ميزان رضايتمندى جانبازان تحت يوشش بيمه}

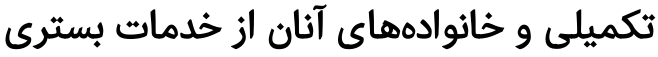

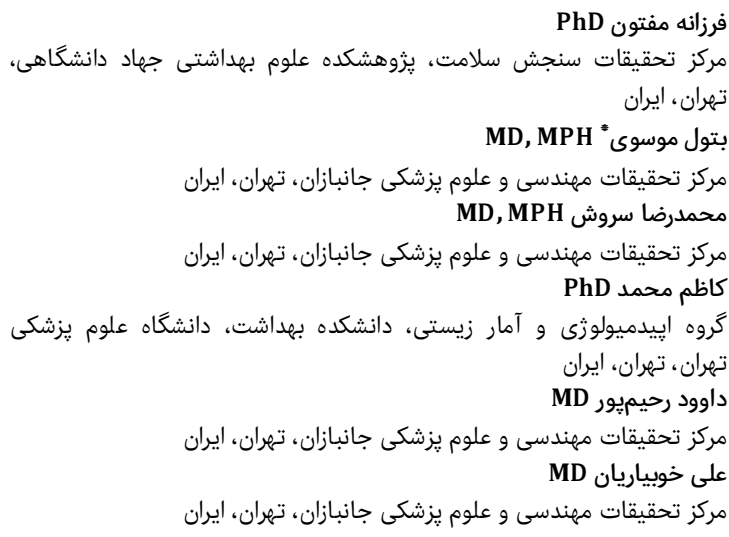

حكيده

أهداف: كيفيت خدمات درمانى و سطح رضايتمندى جانبازان و خانواده آنان از

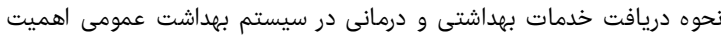

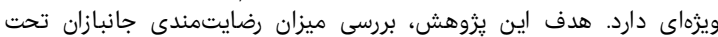

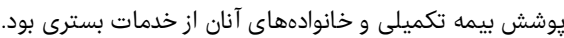

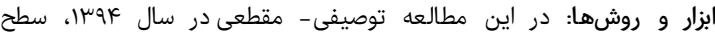

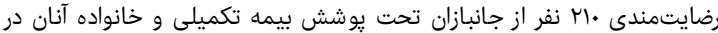

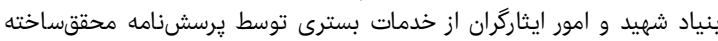

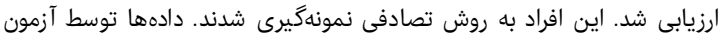

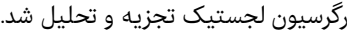

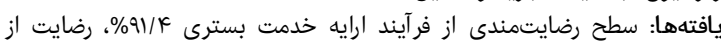

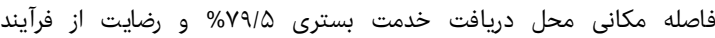

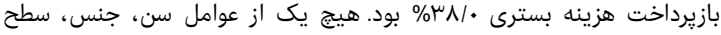

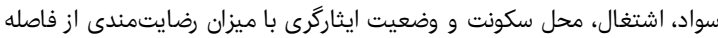

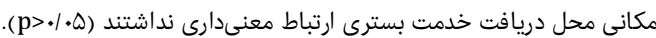

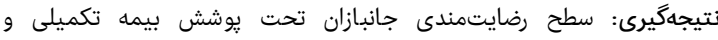

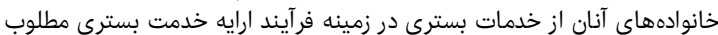

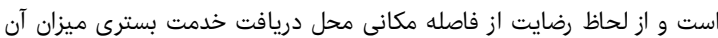
قابل قبول است، در حالى كه رضايت از فرآيند بازيرداخت هزين درينه بسترى مطلوب كليدوازهها: رضايت بيمار، بسترى، جانبازان، بيمارستان، هزينههاى مراقبتهاى بهداشتى

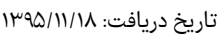

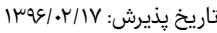

mousavi.b@gmail.com :نويسنده مسئول: تاريخ بذيرث:

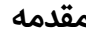

بيمارستان جزء جدايىنايذير تشكيلات يزشكى و اجتماعى است

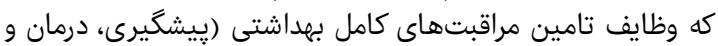

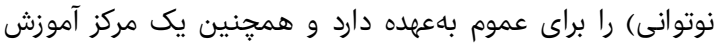

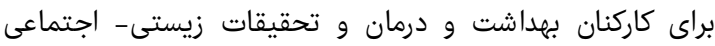

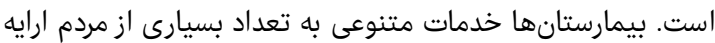

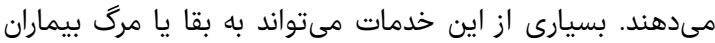

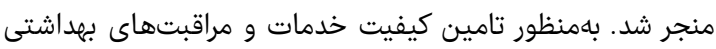

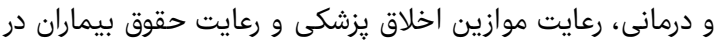

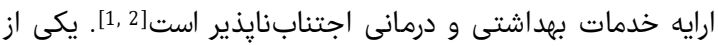

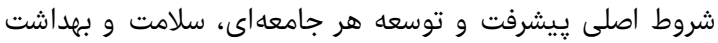

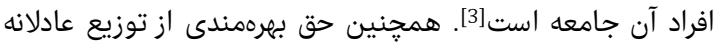

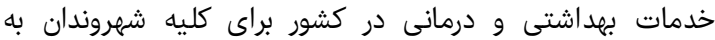
رسميت شناخته شده است. بر همين اساس، تامين مالى خدمات

فصل فامه علمى - يزوهشى طب جانباز 


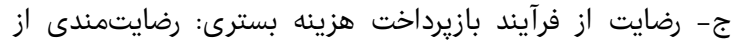
كليه فرآيندهايى است كه مراجعهكننده براى بازيرداخت هزين هزينهاين

$$
\text { خود متحمل شده است. }
$$

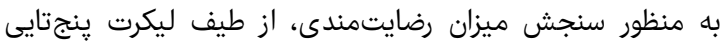

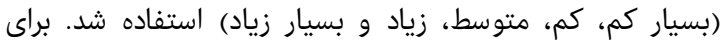

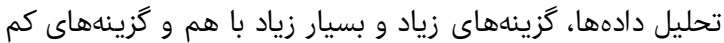

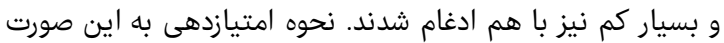

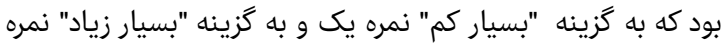

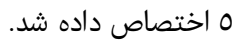

جدول () توزيع فراوانى مطلق و نسبى ويزگى تهاى جمعيت شناختى جانبازان

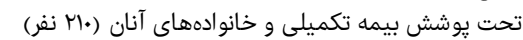

\begin{tabular}{|c|c|c|}
\hline درصد & تعداد & متغيرهاى جمعيت شناختى \\
\hline & & سن (سال) \\
\hline$r / F$ & $\Delta$ & $1-9$ \\
\hline$\Delta / V$ & Ir & $1 .-19$ \\
\hline$r / F$ & $\Delta$ & $r \cdot-r q$ \\
\hline w/A & $\wedge$ & $\mu \cdot-\mu_{q}$ \\
\hline$\mu \Delta / r$ & $V F$ & $k--k q$ \\
\hline$\mu \Delta / r$ & VF & $\Delta \cdot-\Delta Q$ \\
\hline १/• & 19 & 9.-99 \\
\hline \multirow[t]{2}{*}{$\varepsilon / r$} & w & 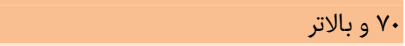 \\
\hline & & جنسيت \\
\hline $9 / 9$ & $1 \pi$. & مرد \\
\hline \multirow[t]{2}{*}{ rN/I } & $\wedge$. & زن \\
\hline & & طول مدت تحصيل (سال) \\
\hline 西/ & rA & كمتر از ا \\
\hline$\mu \Delta / V$ & $\checkmark \Delta$ & $1-\Delta$ \\
\hline$r \cdot / \Delta$ & $k \mu$ & $4-\Lambda$ \\
\hline rM/A & $\Delta \cdot$ & $9-14$ \\
\hline \multirow[t]{2}{*}{ s/V } & If & بالاتر از با \\
\hline & & وضعت اشتغال \\
\hline$\mu k / \mu$ & vr & خانهدار \\
\hline$s / r$ & Ir & دانشجو \\
\hline 1 & r & كارمند \\
\hline 1/9 & k & كاركر \\
\hline $11 \%$ & $r \mu$ & شغل آزاد \\
\hline $1 / 9$ & $k$ & كشاورز \\
\hline$r q / Q$ & sr & بيكار \\
\hline \multirow[t]{2}{*}{$\mid \kappa / \mu$} & $\mu$. & ساير \\
\hline & & محل سكونت \\
\hline $9 \cdot / \Delta$ & 19. & شهر \\
\hline $9 / 0$ & $r$. & روستا \\
\hline$\mu r / k$ & $8 \Lambda$ & وضعيت ايثاركرى هـ و كمتر \\
\hline rm/A & $\Delta$. & جانباز بيشتر از YYQ \\
\hline $\mathrm{kr} / \mathrm{A}$ & $9 r$ & خانو اده جانباز \\
\hline
\end{tabular}

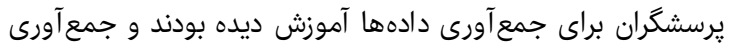

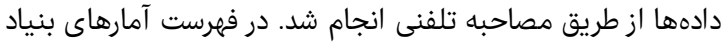

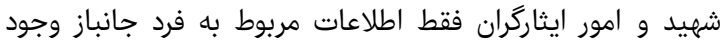

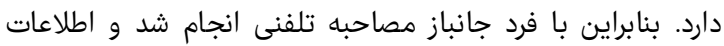

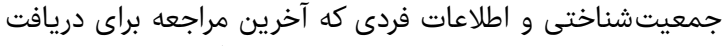

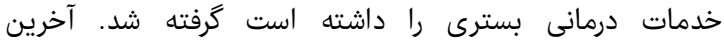

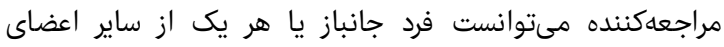

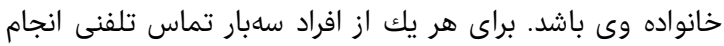

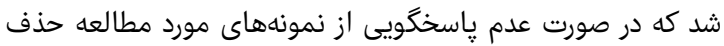

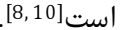

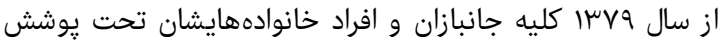

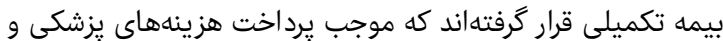

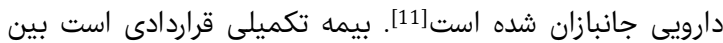

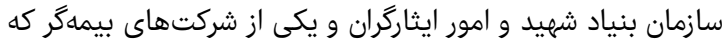

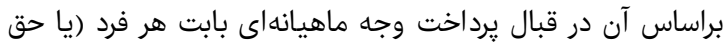

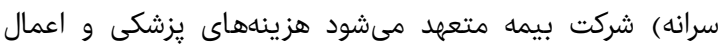

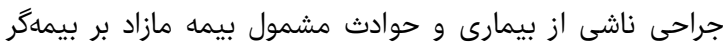

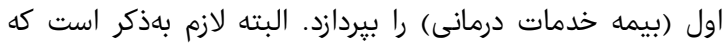

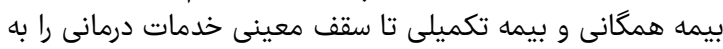

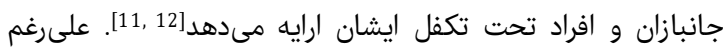

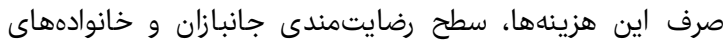

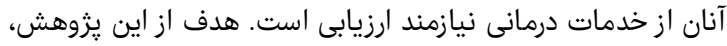

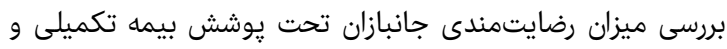

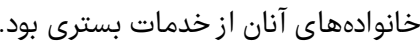

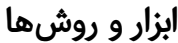

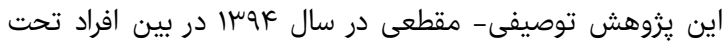

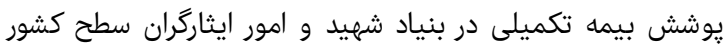

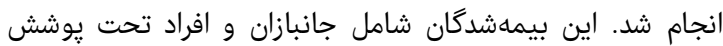

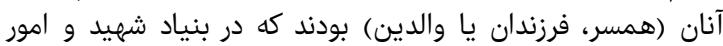

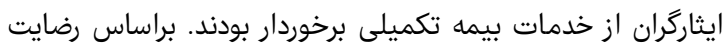

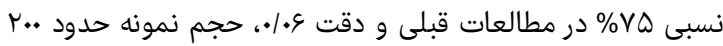

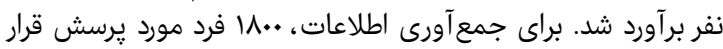

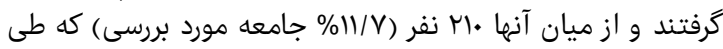

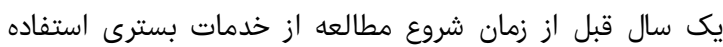

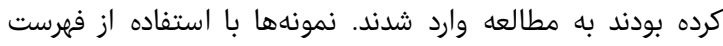

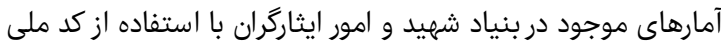

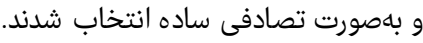

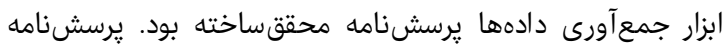

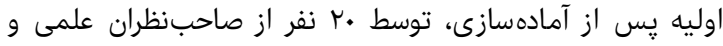

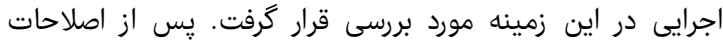

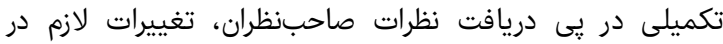

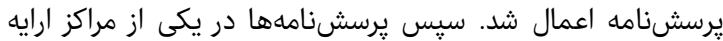

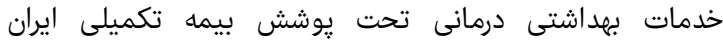

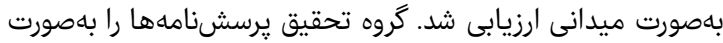

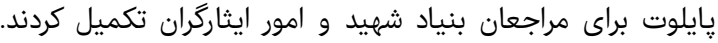

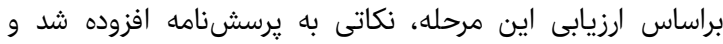

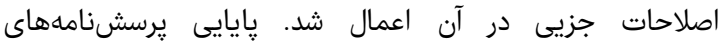
تكميلشده از طريق آزمون آلفاى كرونباخ محاسبه شد كه معادل

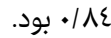
يرسشنامه داراى برد سئوال شامل 10 سئوال در زمينه اطلاعات

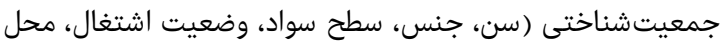

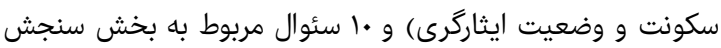

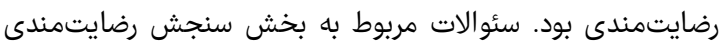

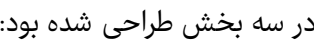

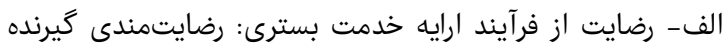

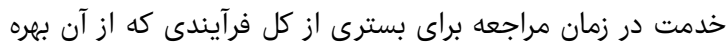
برده است. ب- رضايت از فاصله مكانى محل دريافت خدمت بـاست بسترى: بلمعنى

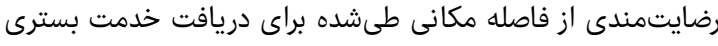


اكثر افراد مورد مطالعه از فرآيند ارايه خدمت بسترى رضايتمندى

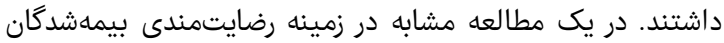

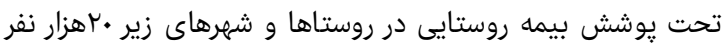

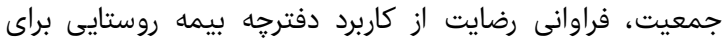

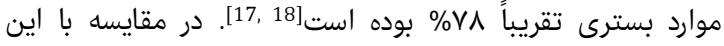

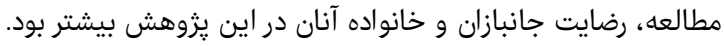

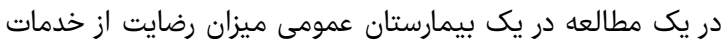

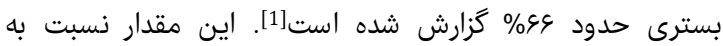

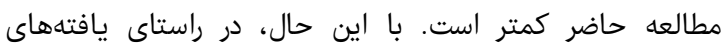

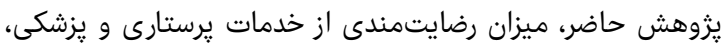

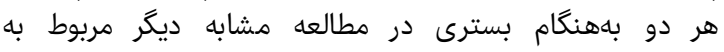

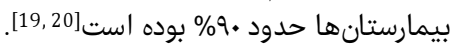

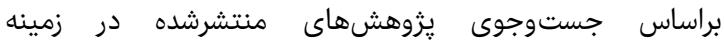

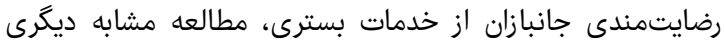

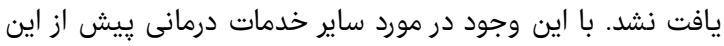

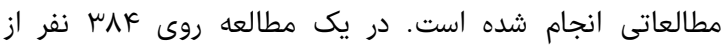

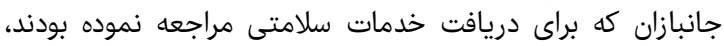

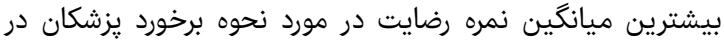

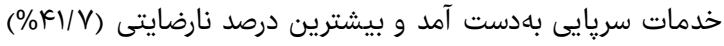

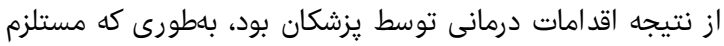

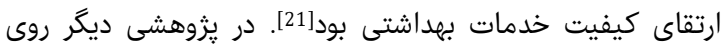

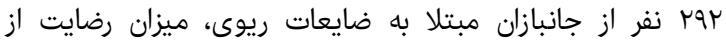

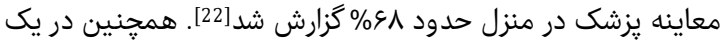

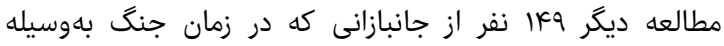

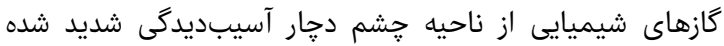

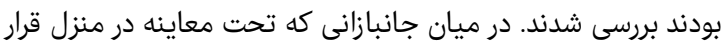

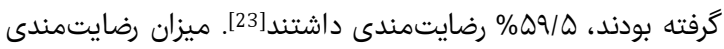

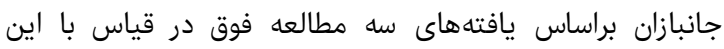
يزوهش كمتر است.

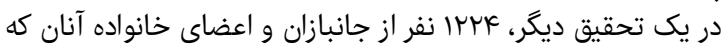

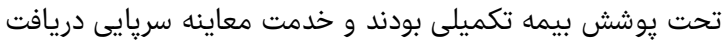

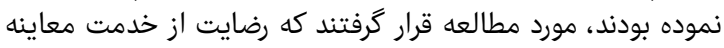

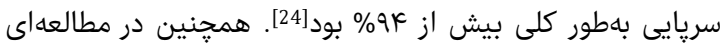

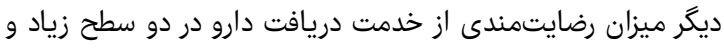

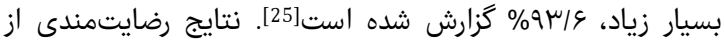

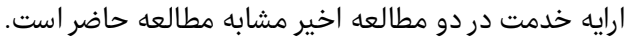

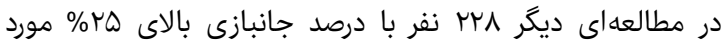

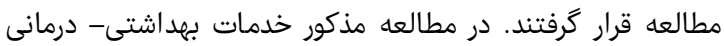

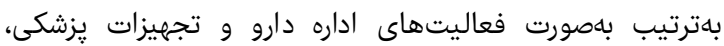

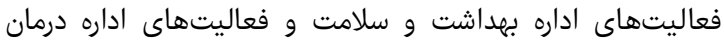

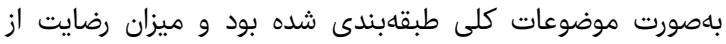

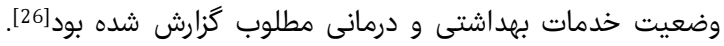

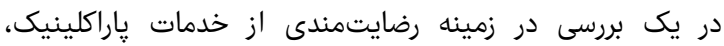

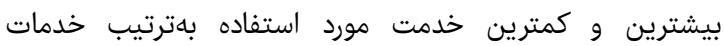

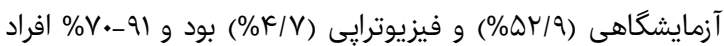

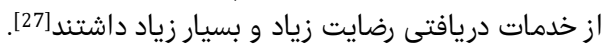

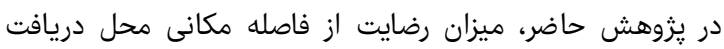

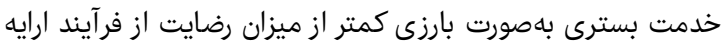

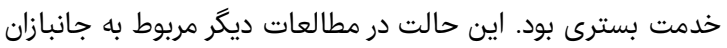

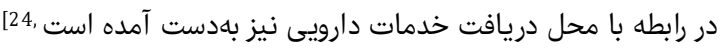

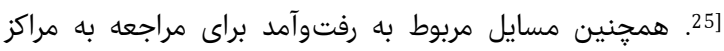

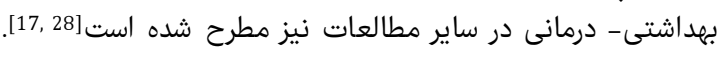

دوره 9، شماره *، بإييز عوسا

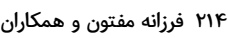

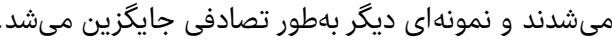

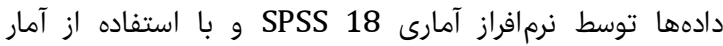

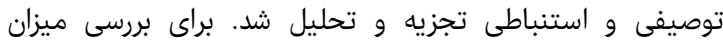

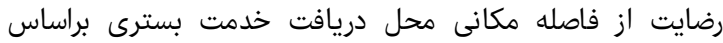

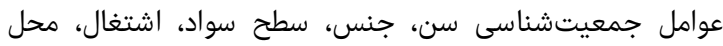

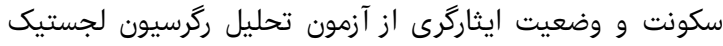

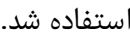

يافتهها

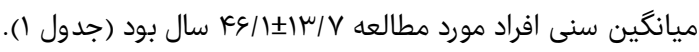

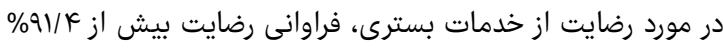

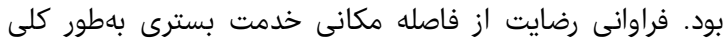

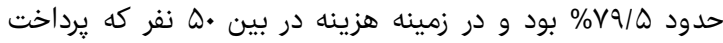

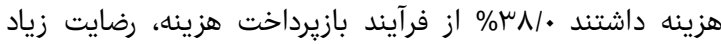

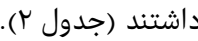

جدول r) توزيع فراوانى مطلق و نسبى (اعداد داخل برانتز درهد هستند) ميزان

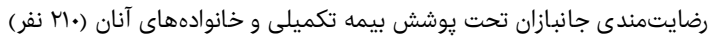

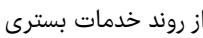

\begin{tabular}{|c|c|c|c|}
\hline 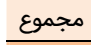 & رضايتمندى زياد & رضايتمندى متوسط & 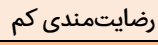 \\
\hline \multirow[b]{2}{*}{ (l..) $\mathrm{Ml}$. } & \multicolumn{3}{|c|}{ عايت از فرآيند ارايه خدمت بسترى } \\
\hline & $(91 / k) 19 r$ & $(Y / 9) \&$ & $(\Delta / V) \mathbb{I r}$ \\
\hline \multicolumn{4}{|c|}{ رضايت از فاصله مكانى محل دريافت خدمت بسترى } \\
\hline (I..) $\mu$ M. & $(\vee १ / \Delta) \backslash \& V$ & $(11 / \cdot) r \mu$ & $(Q / \Delta) r \cdot$ \\
\hline \multicolumn{4}{|c|}{ 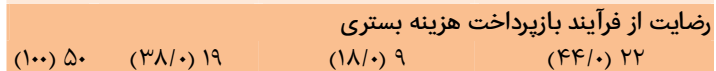 } \\
\hline$(1 \cdot \cdot) \Delta$. & $\left({ }^{\mu} \mathrm{N} / \cdot\right) 19$ & $(\mid \mathrm{N} / \cdot)^{9}$ & $(K F / \cdot) K r$ \\
\hline
\end{tabular}

هيج يك از عوامل سن، جنسيت، سطح سواد، وضعيت اشتغال،

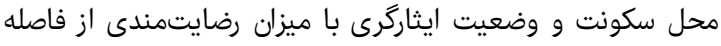

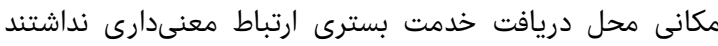

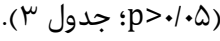

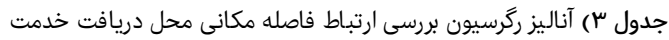

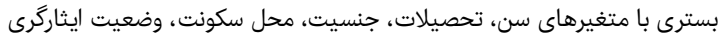

\begin{tabular}{|c|c|c|c|c|}
\hline \multicolumn{5}{|c|}{ و اشتغال } \\
\hline معنىدارى & 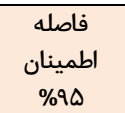 & تعدآورد خطر & B مقدار B & متغيرها \\
\hline. & $. / 9 \vee-1 / 1$. & $1 / \cdot \mu$ &.$/ \cdot \mu$ & سن \\
\hline.$/ 48$ & $. / 9 \mu-1 / 11$ & $1 / \cdot 0$ & .1 .0 & تحصيلات \\
\hline.$/ 99$ & . $|\mu \mu-s| \varphi$. & kr & $I V / \Delta Q$ & جنسيت \\
\hline.$/ \Lambda$ & . & $1 / Y K$ & . & محل سكونت \\
\hline.$/ 99$ & . & Y/IV & $19 / r$. & گروه جانباز \\
\hline . IAt & $\cdot \mid \Lambda V-I \cdot / \mu \lambda$ & $\mu$ & $1 / 1$ & گروه خانواده جانباز \\
\hline .11 & $. / .9-1 / 41$ & س & $-1 / 1$. & وضعيت اشتغال \\
\hline
\end{tabular}

در اين مطالعه ميزان رضايتمندى جانبازان تحت يوشش بيمه

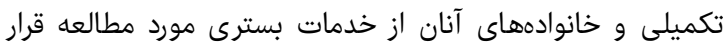

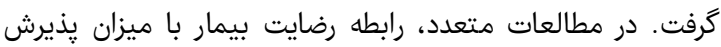

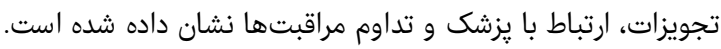

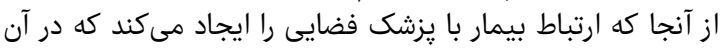

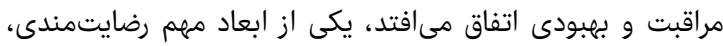

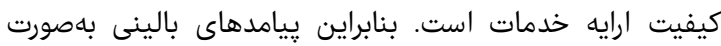

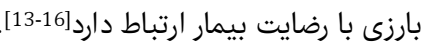

فصل نامه علمى - يزووهشى طب جانباز 


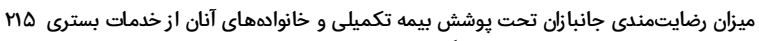

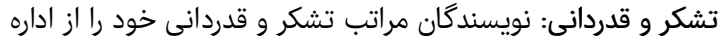

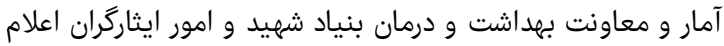

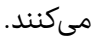
تاييديه اخلاقى: افراد مورد مطالعه با رضايت وارد مطالعه شدند.

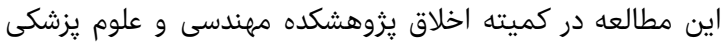
جانبازان، تهران، ايران با شماره

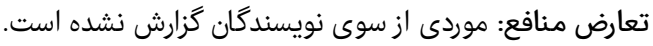

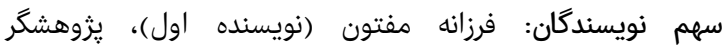

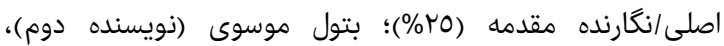

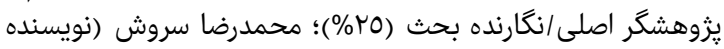

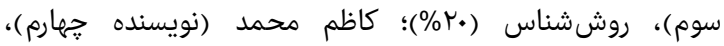

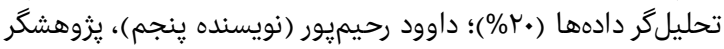

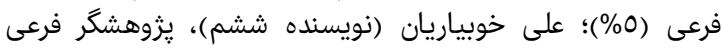

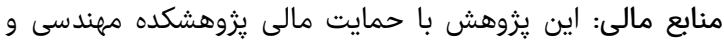

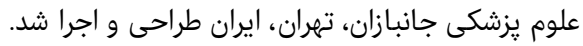

1- Mosadeghrad AM. Essentials of healthcare organization and management. Tehran: Dibagaran Publishing; 2003. p. 534. [Persian]

2- Mosadeghrad AM. Assessment of Patients Satisfaction with Hospital Services in Razi Hospital in Qazvin. J Manag Inform Health. 2005;1(1):28-32. [Persian]

3- Abbaspour A, Davarani SH, Masoumi M, Modirian E, Khateri Sh, Mousavi B, et al. Satisfaction of child victims of landmines with services of veterans and martyr affair foundation (VMAF). Daneshvar. 2015;22(118):43-50. [Persian]

4- Torabi M. Utilization Model of Health Services in Retirees Covered by DANA Insurance [Dissertation]. Tehran: Islamic Azad University; 2003. [Persian]

5- Mosadeghrad AM. The role of participative management (suggestion system) in Shahid Fayazbakhsh Hopital effectiveness and efficiency. J Res Med Sci. 2003;8(3):85-89. [Persian]

6- Applebaum R, Straker J, Georn S. Assessing satisfaction in health and long term care: Practical approaches to hearing the voices of consumers. Salmon Tower Building New York City: Springer Publishing Company; 1999.

7- Avis M, Bond M, Arthur A. Satisfying solutions? a review of some unresolved issues in the measurement of patient satisfaction. J Adv Nurs. 1995;22(2):316-22.

8- Taylor SA, Cronin JJ Jr. Modeling patient satisfaction and service quality. J Health Care Mark. 1994;14(1):3444.

9- Johansoon P, Oleni M, Fridlund B. patient satisfaction with nursing care in the context of health care: A literature study. Scand J Caring Sci. 2002;16(4):337-44.

10- Kravitz R. Patient satisfaction with health care: Critical outcome or trivial pursuit?. J Gen Intern Med. 1998;13(4):280-2.

11- Puor Aghaei M. Medical equipment and quality of life in veterans. First Veterans and Families Scientific Conference, Veterans Institute for Engineering and Medical Sciences. Tehran: Deputy of Cultural Affairs and Sports Affairs of Veterans Affairs; 2003. [Persian]

12- Mousavi B, Maftoon F, Sorush MR, Mohammad K, Rahimpoor D, Khoubyarian A. Satisfaction with outpatient visits in veterans covered by supplementary
نتايج ساير يزوهشها نشانكر اين نكته است كه فاصله مكانى و

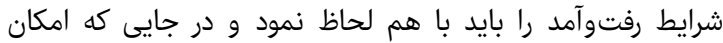
تغيير فاصله مكانى وجود ندارد، بهدنبال راهحلهائ بهائ بهببود شرايط

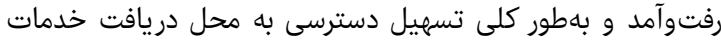

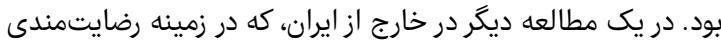

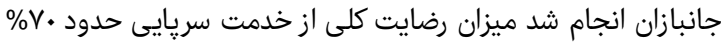

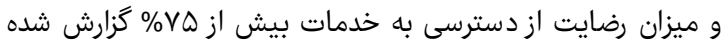

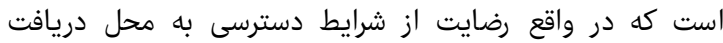

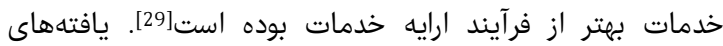
مطالعه فوق برخلاف نتايج مطالعه حاضر است إندات

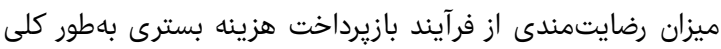

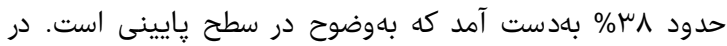

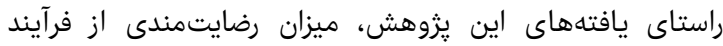

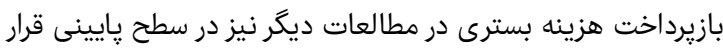

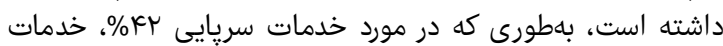

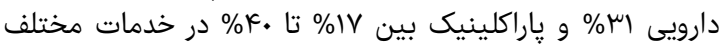

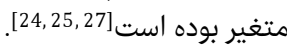

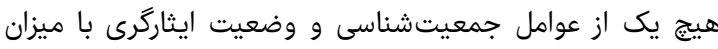

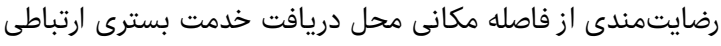

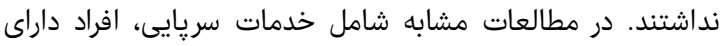

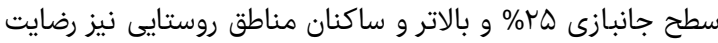

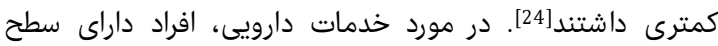

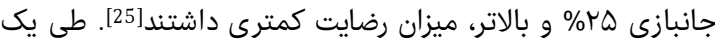

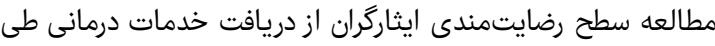

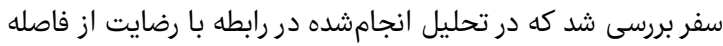

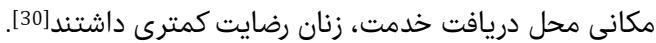

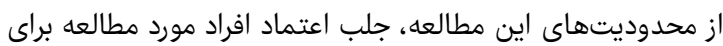

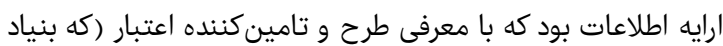

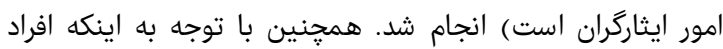

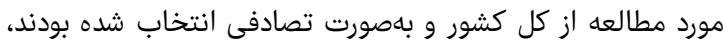

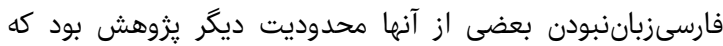

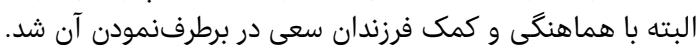

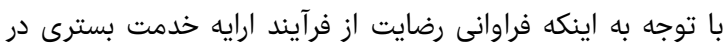

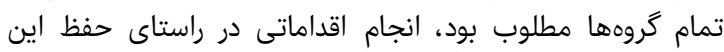

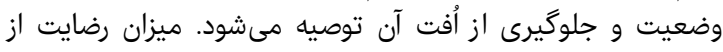

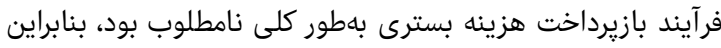

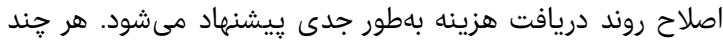

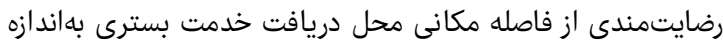

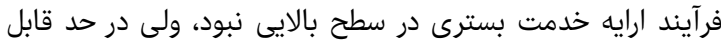

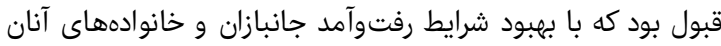

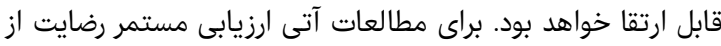

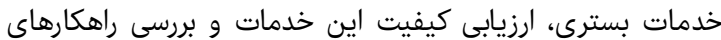

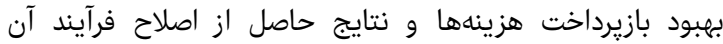
ييشنهاد مى شود. رضايتمندى جانبازان تحت يوشش تِيرى بيمه تكميلى و خانوادههاى

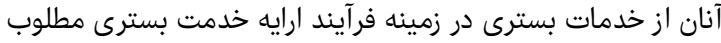

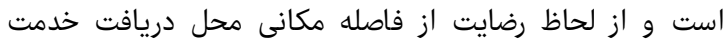
بسترى در سطح قابل قبولى قرار دارد، در حالى كه رضايت رضايت از إن فرآيند بازيرداخت هزينه بسترى مطلوب نيست فئ. 
Med Sci. 2006;4(1):41-8. [Persian]

22- Mousavi B, Jafari F, Davati A, Soroush MR, Narenjkar J. Health services satisfaction among chemical warfare survivors: A national survey from Iran. Iran J War Public Health. 2009;2(1):1-5. [Persian]

23- Soroush MR, Mousavi B, Ganjparvar Z. Health care service satisfaction among chemical warfare survivors with severe ophthalmogic complications. Iran J War Public Health. 2008;1(1):12-21. [Persian]

24- Mousavi B, Maftoon F, Soroush MR, Mohammad K, Rahimpoor D, Khoubyarian A. Satisfaction with outpatient visits in veterans covered by supplementary health insurance. Pyesh. 2017;16(2):142-9. [Persian] 25- Mousavi B, Maftoon F, Mohammad K, Soroush MR, Asgari M. Satisfaction with supplemental health insurance and pharmaceutical care among veterans and their families. Pyesh. 2016;15(6):609-17. [Persian]

26- Jandaghi Gh, Tabarsa Gh, Heidari $H$, Heidari $F$. Satisfaction of veterans from health services and their impact on mental well-being. Iran J War Public Health. 2010;3(9):30-6. [Persian]

27- Maftoon F, Mousavi B, Mohammad K, Soroush MR, Ganjparvar Z. satisfaction of martyrs' families of supplemental insurance in receiving para-clinical services. Iran J War Public Health. 2015;7(4):225-32. [Persian]

28- Newman RD, Nyangezi JM, Machobo F, Muiser J. Satisfaction with outpatient health care services in manica province, Mozambique. Health Policy Plan. 1998;13(2):174-80.

29- Wright SM, Craig T, Campbell S, Schaefer J, Humble Ch. Patient Satisfaction of Female and Male Users of Veterans Health Administration Services. J Gen Intern Med. 2006;21(Suppl 3): S26-32.

30- Soroush MR, Mousavi B, Maftoon F, Mohammad K, Ganjparvar Z. Satisfaction of supplementary insurance and medical services during trips in war survivors and their families. Int J Travel Med Glob Heath. 2016;4(2):658.
- فرزانه مفتون و همكاران M M

health insurance. J Iran Institut Health Sci Res. 2017;16(2):142-9. [Persian]

13- Williams S, Dale J. Doctor-patients communication and patient satisfaction: A review. Fam Pract Int J. 1998;15(5):480-92.

14- Winefield HR, Murrell TG, Clifford J. Process and outcomes in general practice consultations: Problems in defining high quality care. Soc Sci Med. 1995;41(7):96975 .

15- Loblaw DA, Bezjak A, Bunston T. Development and testing of a visit-specific patient satisfaction questionnaire: The Princess Margaret hospital satisfaction with doctor questionnaire. J Clin Oncol. 1999;17(6):1931-8.

16- Kane RL, Maciejewski M, Finch M. The relationship of patient satisfaction with care and clinical outcomes. Med Care. 1997;35(7):714-30.

17- Maftoon F, Mohannad K, Montazeri A, Farzadi F, Aeenparast A. Care receivers satisfaction in family physician and referral system: A national study from Iran. J Int Res Med Pharm Sci. 2016;7(4):180-6.

18- Maftoon F, Montazeri A, Farzadi F, Jahangiri K, Aeenparast A, Mohammad K, et al. Survey of satisfaction of rural insured covered by the health insurance organization and providers in the referral implication plan and family physician. Tehran: Health Insurance Organization; 2009. [Persian]

19- Hajian K. Satisfaction rate of hospitalized patients from the provision of health services in Shahid Beheshti and Yahya Nejad hospitals in Babol. J Babol Univ Med Sci. 2008;9(2):51-60. [Persian]

20- Soleimani V, Ghalyabtab F. Inpatient satisfaction: Medical services delivery in social security hospital (Chamran) in Saveh. J Hospital. 2006;6(1-2):9-10. [Persian]

21- Shojaei Baghini H, Nakhaei N, Aghaei Afshar M. Satisfaction of janbazan and azadegan referring health center of janbazan foundation of Kerman City to Rcceive Medical Services in the Year 2003-2004. J Rafsanjan Univ 